\title{
Azathiopine for the Treatment of Extensive Forms of Alopecia Areata: A Systematic Review
}

\author{
Val Constantine S. Cua, ${ }^{1}$ Juan Paolo David S. Villena, ${ }^{1}$ Felix Paolo J. Lizarondo ${ }^{1}$ and Claudine Yap-Silva ${ }^{2}$ \\ ${ }^{1}$ Section of Dermatology, Department of Medicine, Philippine General Hospital, University of the Philippines Manila \\ ${ }^{2}$ Section of Dermatology, Department of Medicine, College of Medicine and Philippine General Hospital, University of the Philippines Manila
}

\begin{abstract}
Background. Alopecia areata (AA) is an autoimmune hair disorder, with the clinical variants ophiasis and extensive variants AA totalis and universalis, having poor response to therapy. Oral steroids are used to treat the severe variants, requiring prolonged therapy, which leads to side effects while discontinuation leads to high relapse rate. Azathioprine is a steroid-sparing agent for the severe AA forms.
\end{abstract}

Objective. To review the current evidence on the therapeutic efficacy and adverse effects of azathioprine for severe forms of alopecia areata

Methods. Published articles utilizing azathioprine for alopecia areata were obtained until July 2018 from PubMed, MEDLINE, Cochrane Library, TRIP database, HERDIN, and Google Scholar.

Results. Seven articles underwent a full-length review. Clinical variants include patchy, diffuse, steroid-resistant, reticulate, totalis, universalis, ophiasis, and sisaipho. Doses ranged from 2 to $2.5 \mathrm{mg} / \mathrm{kg} / \mathrm{day}$ or weekly $5 \mathrm{mg} / \mathrm{kg}$ pulse therapy. Initial response ranged from 6 to 12 weeks, with almost complete resolution by 32 weeks. Response was sustained for 6 months upon discontinuation, with only $14 \%$ relapsing at 2.5 months. Adverse effects were gastrointestinal discomfort, elevated liver function tests, and myelosuppression.

Conclusion. There is emerging evidence on the efficacy and safety of azathioprine for the treatment of extensive forms of alopecia areata. Randomized-controlled trials are needed to evaluate its efficacy.

Key Words: Alopecia Areata, Totalis, Universalis, Azathioprine, Systematic Review

\section{INTRODUCTION}

E-poster presented at the $41^{\text {st }}$ Annual Convention of the Philippine Dermatological Society, November 7-9, 2018, EDSA Shangri-La, Mandaluyong City, Philippines

Finalist for the digital poster presented at the $19^{\text {th }}$ Dubai World Dermatology and Laser Conference and Exhibition, March 18-20, 2019, Dubai International Convention and Exhibition Centre, Dubai World Trade Centre, Dubai, United Arab Emirates

Oral presentation at the $1^{\text {th }}$ Dubai World Dermatology and Laser Conference and Exhibition, March 18-20, 2019, Dubai International Convention and Exhibition Centre, Dubai World Trade Centre, Dubai, United Arab Emirates

Corresponding author: Val Constantine S. Cua, MD

Section of Dermatology

Department of Medicine

Philippine General Hospital,

University of the Philippines Manila

Taft Avenue, Manila 1000, Philippines

Telephone: +632 5548400 local 5105/5106

Email:vscua@up.edu.ph
Alopecia areata (AA) is a non-scarring hair disorder that presents with well demarcated solitary or multiple non-cicatricial round or oval hairless patches with the characteristic finding of "exclamation mark hairs", near the advancing margins. ${ }^{1}$ It has been hypothesized to be an organ-specific autoimmune disease that involves the breakdown of the hair follicle immune privilege ${ }^{2}$ and the production of autoantigens through the inappropriate presentation of antigens to the immune system during normal hair follicle cycling. ${ }^{3}$ Histopathologically, this is illustrated by the classic peribulbar lymphocytic infiltrates (swarm of bees) preferentially targeting the anagen stage follicles. ${ }^{4}$

Alopecia areata can be classified based on its presentation ${ }^{3}$ : partial loss of scalp hair (patchy alopecia areata), loss of all scalp hair (alopecia totalis), or loss of all body hair (alopecia universalis). Other variants which are relatively uncommon include: ophiasis which is a band-like hair loss in the parieto-temporo-occipital area; ophiasis 
inversus or sisaipho which presents as band-like hair loss in the fronto-parieto-temporal area; diffuse thinning over a part or all over the scalp; and reticular patches of hair loss. ${ }^{3}$ In a study done in Singapore ${ }^{5}$, the authors noted that around $58 \%$ of the study population with AA presented with less than 50\% scalp involvement, $32.4 \%$ with $50-99 \%$ scalp involvement, $7.8 \%$ with alopecia totalis (AT) and $1.8 \%$ with alopecia universalis (AU). In India, only $7.2 \%$ of the AA population studied presented with the ophiasis pattern of alopecia areata. ${ }^{6}$

The natural course of AA is unpredictable. Albeit not having a preventive treatment for alopecia areata, the hair follicles in the said disorder are preserved, maintaining the potential for hair growth recovery even in longstanding disease. ${ }^{7}$ Spontaneous remission (34-50\%) within one year has been noted for small number of patchy $\mathrm{AA}^{7}$, with $31.2 \%$ occurring within 6 months. ${ }^{8}$ The patient may still relapse $(85.8 \%)$ and have focal alopecic patches after remission. For some, the condition may progress to more extensive forms of alopecia areata (14-25\%), and only less than 10\% recover from AT and AU. ${ }^{8}$

Current treatments are directed towards halting disease activity. In the latest 2012 guideline for the management of alopecia areata by the British Association of Dermatologists, only two treatments had a strength recommendation $\mathrm{C}$ (a body of evidence in high-quality systematic reviews, case-control, or cohort studies): potent topical steroid and intralesional corticosteroid for limited patchy hair loss; and contact immunotherapy for extensive patchy hair loss, AT and AU. Reports on the success rate for topical steroid vary from 28.5 to $61 \%$, with the relapse rate as high as $63 \% .{ }^{9,10}$ For intralesional steroids, the success rate ranges from 60-75\%, with the effect lasting for 6-9 months in around $60 \%$ of patients. ${ }^{11,12}$ It is, however, only recommended for lesions fewer than 5 patches of less than $3 \mathrm{~cm}$ in diameter. ${ }^{11}$ A systematic review of steroid pulse therapies for AA which noted complete response was achieved in $43 \%$ of the total study population, with a relapse rate of $17 \%$ for the responders. ${ }^{13}$ Two studies, however, noted that steroid pulse therapy is not recommended for AT and AU. ${ }^{14,15}$ Adverse effects noted for corticosteroid therapy are as follows: for topical therapy, folliculitis ${ }^{10}$, telangiectasia ${ }^{3}$, skin atrophy ${ }^{11}$, and hypopigmentation ${ }^{16}$; for the intralesional approach, skin atrophy, and increased risk for developing cataract and raised intraocular pressure when used near the eyes ${ }^{17}$; and for systemic treatment, weight gain, Cushing syndrome, striae, irritability ${ }^{18}$, arthralgia, myalgia, malaise, hypertension, hyperglycemia, increased susceptibility to infection, behavioral disturbances, rebound flare-up of the treated disease $^{19}$, anaphylaxis ${ }^{20,21}$, decrease in bone mineral density ${ }^{22}$, acneiform eruption, abdominal discomfort, weakness, dysmenorrhea, and adrenocortical suppression. ${ }^{23}$

Other treatments noted in literature were phototherapy and laser, cryotherapy, janus kinase (JAK) inhibitors, immunomodulators, stem cell educator therapy, statins, and prostaglandin analogues ${ }^{12}$; all of which have not been extensively studied for severe forms of alopecia areata. Indeed, the therapeutic challenge posed by extensive AA is either the lack of efficacy or the side effects of current available options. ${ }^{24}$ Furthermore, there is a scarcity of FDAapproved systemic therapy due to the lack of randomized controlled trials for efficacy and safety. Recent researches ${ }^{25-27}$, however, have focused on immunosuppressants like azathioprine as an alternative to corticosteroid therapy to circumvent the side effects of the latter.

Azathioprine is a synthetic purine analog that functions as a purine antagonist. It is derived from 6-mercaptopurine by the addition of an imidazole ring to protect the product from immediate metabolic degradation. ${ }^{28}$ Metabolism of the drug is achieved by three enzymes. Xanthine oxidase $(\mathrm{XO})$ and thiopurine s-methyltransferase (TPMT) convert mercaptopurine to inactive metabolites (6-thiouric acid and 6-methyl-mercaptopurine, respectively) while hypoxanthine phosphoribosyl transferase (HPRT) converts the drug to the active metabolite, 6-thioguanine nucleotide. ${ }^{29}$ The active metabolite disrupts the function of the endogenous purines by interfering with purine synthesis and metabolism. ${ }^{29}$ This contributes to its specificity in targeting lymphocytes since the latter lack a salvage pathway to synthesize new purines. ${ }^{30} \mathrm{It}$ is through this mechanism of action that azathioprine is able to target the etiology of autoimmune diseases such as alopecia areata. Recently, its role as an immunomodulator is being investigated as an alternative steroid-sparing treatment for alopecia areata, particularly the severe and extensive types.

The primary objective of this review was to assess the efficacy of azathioprine in the treatment of alopecia areata, particularly the severe types: alopecia totalis, alopecia universalis, and ophiasis. The secondary objective of this review was to determine the adverse effects of azathioprine in the treatment of alopecia areata.

\section{METHODS}

Literature search was done on the following electronic databases: PubMed, MEDLINE, Cochrane Library, Trip Database, HERDIN (Health Research and Development Information Network) and Google Scholar from the date of creation until July 26, 2018. The following keywords were used: "alopecia areata", "alopecia", and "azathioprine".

Observational and interventional studies that utilized azathioprine as a form of treatment for all types of alopecia areata were included in this review. Initial screening was done using titles and abstracts. Included studies then underwent full-text evaluation.

A table for data extraction was filled out with the following information: authors, study design, year and the country where the study was done, sample sizes for the alopecia areata, mean age and sex of the participants, type of alopecia observed, co-morbidities (for the case reports 
with primary autoimmune disease reported), inclusion and exclusion criteria (for interventional studies), dosage of azathioprine, other medication taken, treatment response assessment, treatment duration, time to initial response, overall response to treatment and the time duration for this, sustained response duration, time to relapse, rate of relapse (if applicable), adverse effects and authors' conclusion.

\section{RESULTS}

The electronic search through PubMed, MEDLINE, Cochrane Library, Trip Database, HERDIN (Health Research and Development Information Network) and Google Scholar generated 12 records, with 4 duplicates noted. The remaining 8 articles were then screened using the title and abstract. One clinical trial article was not inluded due to inability to obtain the full text. Only 7 full text articles met the selection criteria and underwent qualitative analysis. The study flow diagram in this review is shown in Figure 1.

The seven articles were then categorized into observational studies and interventional studies. For the observational studies, 3 case reports and 1 case series were included and analyzed. For the interventional studies, 2 pre-post studies and 1 controlled clinical trial were evaluated. A pre-post study is a type of experimental design that measures the occurrence of an outcome before and after an intervention is implemented. ${ }^{31}$ No randomized controlled trial was found.

Table 1 shows the qualitative data extracted from the observational studies. The mean age of the patients in the case reports was 30 years old. All patients were diagnosed with a primary autoimmune disorder prior to developing their respective form of extensive alopecia areata. Due to the nature of the primary disorders, all of the patients were started on systemic corticosteroid therapy ${ }^{32,35}$ while patient in one case report ${ }^{33}$ was treated with a series of immunosuppressant before starting with azathioprine. The mean age for the case series was 34.8 years old, and all of them had steroid-resistant alopecia areata, prompting the authors to search for other treatment modalities.
The types of alopecia areata noted for the observational studies were alopecia totalis, alopecia universalis, and steroid resistant AA. The dose of azathioprine per day was approximately 50 to $100 \mathrm{mg}$ per day. Treatment response was measured by hair regrowth and the treatment duration ranged from 1 to 5 years. Initial response was noted as early as 6 weeks while almost complete resolution of the alopecia was recorded by the $8^{\text {th }}$ month. Only Goddard and colleagues ${ }^{32}$ noted sustained response up to 4 months after azathioprine cessation. Only Bala and Chong ${ }^{34}$ noted adverse reaction to azathioprine namely, neutropenia, anemia, fatigue, photosensitivity, headaches, infection, lethargy, and nausea. The neutropenia was argued to be autoimmune in nature by the authors and not related to azathioprine. Overall, the authors in the observational studies concluded that their respective reports were the first ones to note positive response of alopecia areata to azathioprine. Goddard and colleagues ${ }^{32}$ expressed concern that prolonged azathioprine therapy may be needed to provide continuous response in alopecia areata. Vivehanantha and Velangi $i^{33}$ tested azathioprine on two separate occasions for their alopecic patient and noted positive hair regrowth on those said instances, thereby concluding that the response was unlikely due to chance. The conclusion of the case series ${ }^{34}$ was that azathioprine is an effective second line treatment for steroid resistant AA.

Table 2 shows the qualitative data extracted from the 2 pre-post studies. The sample size and the mean age group for these studies were relatively similar. The types of alopecia addressed in these studies were those of nonscarring alopecia covering more than $20 \%$ of the scalp, and that with a duration of more than 6 months, AT, AU, and AU that were not responding to oral corticosteroids and the topical immunomodulator diphenocyprone. Farshi and colleagues ${ }^{25}$ also stated that participants should have been free of any alopecia treatment for at least a month and that no other drugs would be used that can influence hair growth during the study. Pregnant and lactating women were excluded from the study.

The dose of azathioprine was maintained at least 100 mg per day for both studies. Farshi and colleagues ${ }^{25}$ used an objective scale to measure hair regrowth called Severity of

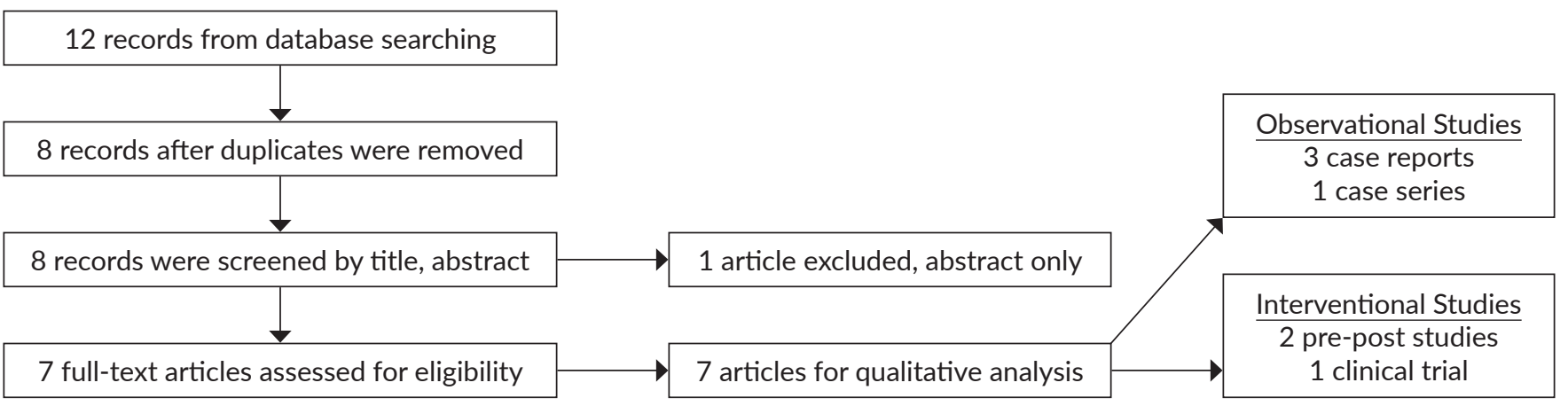

Figure 1. Study flow diagram. 


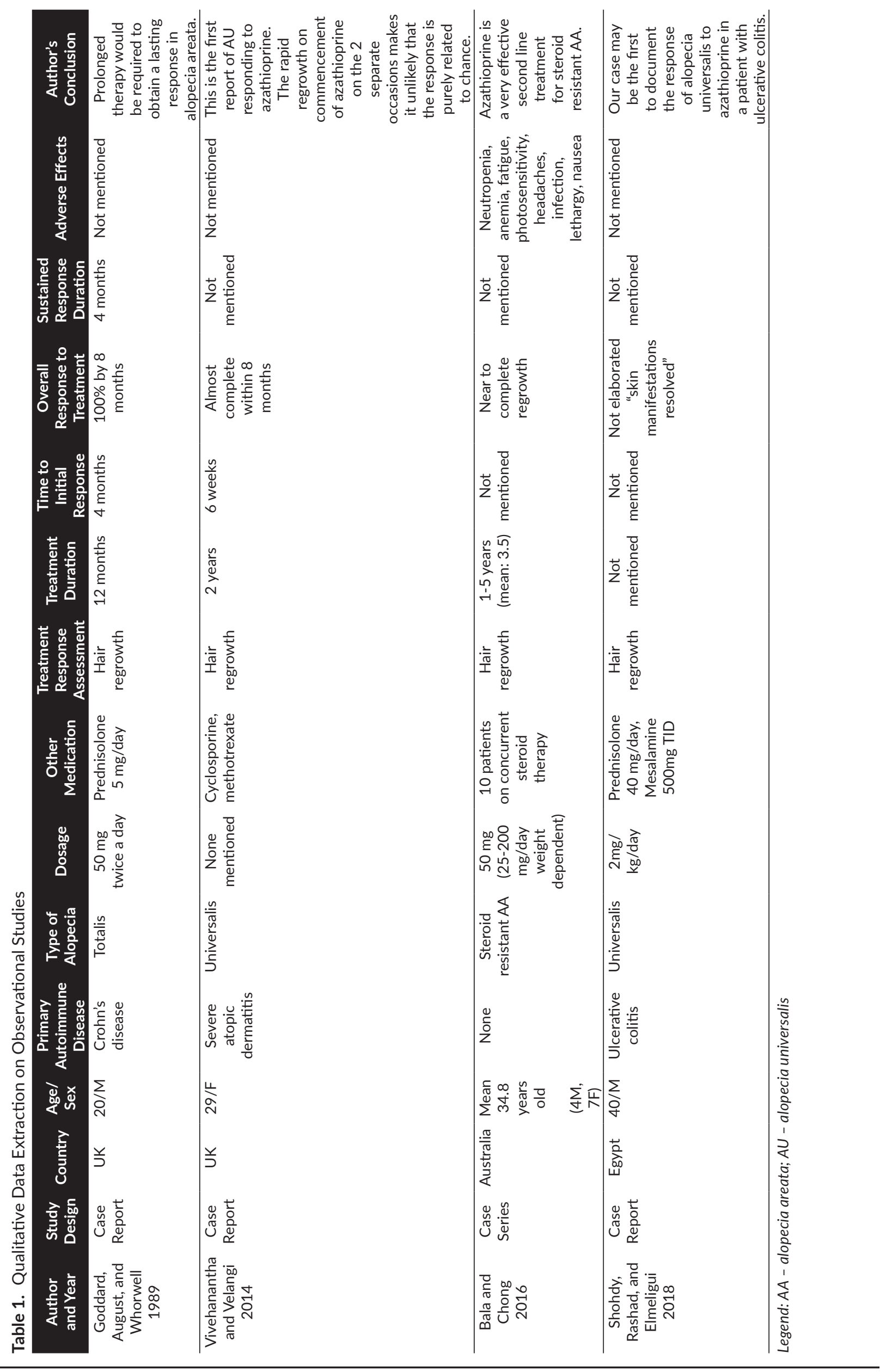




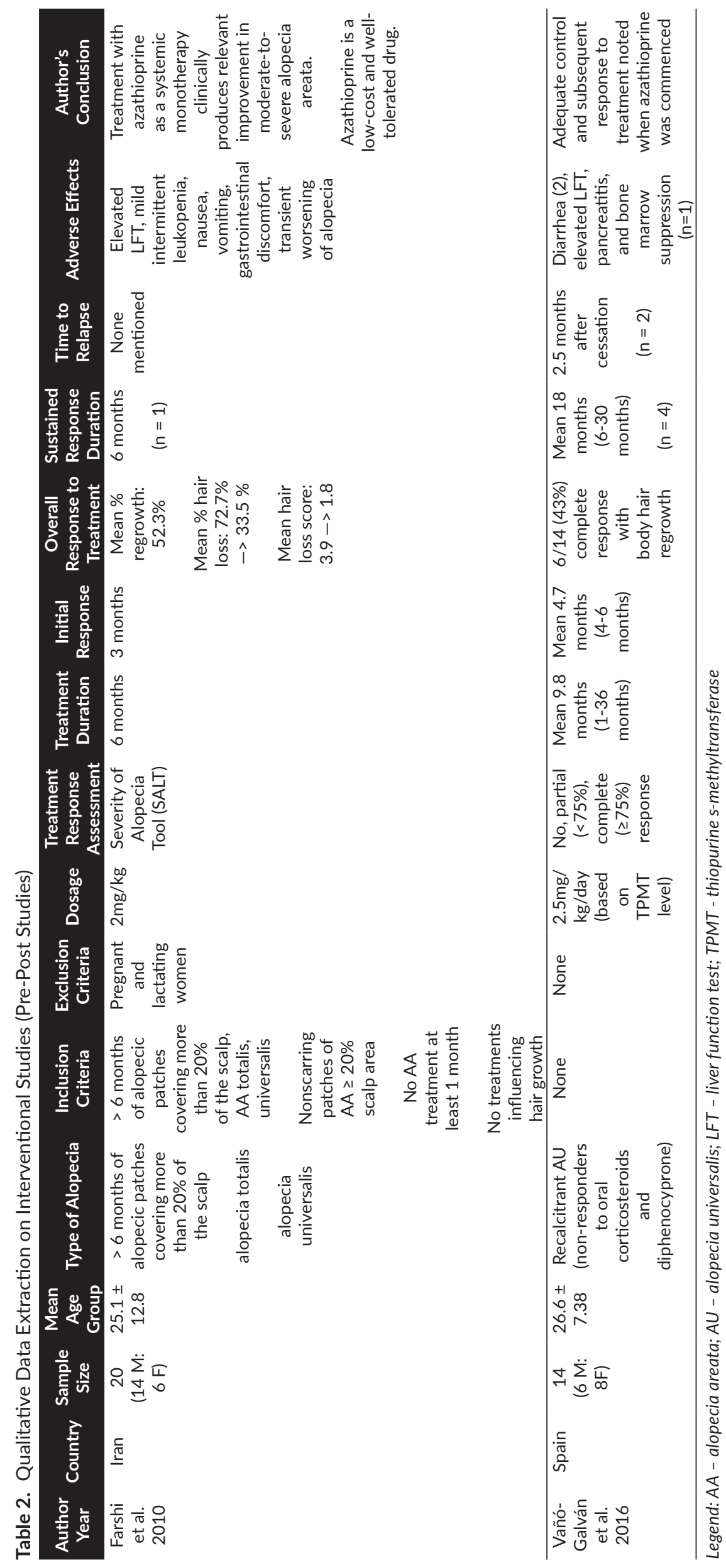


Alopecia Toll (SALT) while Vañó-Galván and colleagues ${ }^{36}$ categorized the outcomes as none, partial $(<75 \%$ hair regrowth) and complete ( $\geq 75 \%$ regrowth).

The SALT score is an objective parameter developed by the National Alopecia Areata Foundation working committee to measure the response of alopecic areas to treatment. ${ }^{37}$ Percentage of hair loss is measured in each of 4 areas of the scalp (40\% vertex, 18\% right profile, $18 \%$ left profile, $24 \%$ posterior), and the sum of all four areas equals the SALT score. Based on the composite score, the patient may be categorized into the following SALT subclasses: $\mathrm{S} 0=$ no hair loss, $\mathrm{S} 1=<25 \%$ scalp hair loss, $\mathrm{S} 2=26-50 \%$ scalp hair loss, $\mathrm{S} 3=51-75 \%$ scalp hair loss, $\mathrm{S} 4 \mathrm{a}=76-95 \%$ scalp hair loss, S4b $=96-99 \%$ scalp hair loss, and S5 = $100 \%$ scalp hair loss. Hair regrowth rate is assessed based on the change in SALT score from baseline. The following are the categories of treatment response using the SALT scoring system: $\mathrm{A} 0=$ no change or further loss, $\mathrm{A} 1=1-24 \%$ regrowth, $\mathrm{A} 2=25-49 \%$ regrowth, $\mathrm{A} 3=50-74 \%$ regrowth, A $4=75-99 \%$ regrowth, A5 $=100 \%$ regrowth.

Treatment duration lasted at least 6 months. Initial response was noted as early as the third month of treatment..$^{25}$ Farshi and colleagues ${ }^{25}$ reported a mean $52.3 \%$ hair regrowth, and a decrease in the percentage of hair loss of $39.2 \%$. The mean hair loss score also decreased by 2.1. On the other hand, Vañó-Galván and colleagues ${ }^{36}$ reported $43 \%$ complete response in their sample population, including body hair regrowth. Most response was sustained for at least 6 months after azathioprine cessation. VañóGalván and colleagues ${ }^{36}$ reported only 2 patients relapsing as early as 2.5 months. Common adverse effects noted were GI symptoms such as discomfort, nausea, vomiting, diarrhea, elevated liver function tests, pancreatitis, mild intermittent leukopenia, bone marrow suppression, and transient worsening of alopecia. The authors concluded that azathioprine is a low-cost, generally well-tolerated drug that has relatively good safety profile, and can provide adequate control of the hair disorder on commencement of treatment.

Table 3 shows the qualitative data extracted from the clinical trial. This study compared the response of alopecia areata to the usual corticosteroid treatment and azathioprine using pulse therapy for both. The population size was equal for both groups, and the mean age of the participants were similar. The types of alopecia included in the study composed mostly of patchy AA, and a few diffuse AA, AU, AT, reticulate, ophiasis, sisaipho and diffuse pattern though the percentage of the latter groups were not stated. The authors compared weekly $5 \mathrm{mg} / \mathrm{kg}$ oral azathioprine and two consecutive days per week of $1 \mathrm{mg} / 10 \mathrm{~kg}$ oral betamethasone pulse therapy for 6 months. The SALT score was used as measurement for treatment response. Based on the change in SALT scores, the patients were categorized based on the following: poor $(<25 \%$ reduction in the baseline SALT score), moderate (26-50\%), good (51-75\%) and excellent response (> 75\%). The mean SALT score at baseline for the weekly azathioprine pulse (WAP) group was $27.85 \pm 23.47$ while that of the betamethasone oral mini pulse (BOMP) group was $34.37 \pm 25.53$. At the end of the study, the mean SALT scores were $0.9 \pm 3.85$ for the former, and $4.01 \pm$ 8.24 for the latter. The large mean differences showed that both therapies proved to be effective in treating alopecia areata. In both groups, the authors noted gradual reduction in mean SALT scores at each follow-up visits. At $6^{\text {th }}$ week, there was $67 \%$ and $70 \%$ reduction in mean SALT score for the BOMP and WAP groups, respectively. Significant reduction from the baseline SALT score was noted at the end of 18 weeks and 24 weeks in the WAP group. For the WAP group, most patients had good to excellent response while the BOMP group had poor and moderate responders. Quicker response was noted in the WAP group, but most patients in both treatment groups were still able to achieve $\mathrm{SALT}_{75}$, which is a $75 \%$ reduction in the base line SALT score, at the end of the clinical trial. No relapse was observed in the WAP group after 6 months of azathioprine cessation while $8.3 \%$ of the patients showed relapse in the BOMP group. Common adverse effects noted for azathioprine were diarrhea, nausea, vomiting, epigastric pain, headache and drowsiness while BOMP patients experienced acneiform eruption, nausea, and headache. One patient in the latter group started developing cushingoid features, and was immediately transferred to the WAP group. This patient showed excellent response thereafter according to the authors. Sonare and colleagues ${ }^{27}$ concluded that weekly azathioprine pulse therapy may be considered as effective as oral betamethasone pulse therapy with lesser side effects.

Table 4 summarizes the common adverse effects noted with azathioprine intake. Only 4 out of the 7 studies documented unfavorable experiences from the patients. The most common side effect was nausea and vomiting $(5.33 \%$ or 4 out of 75 patients), followed by diarrhea and mild intermittent leukopenia ( $4 \%$ or 3 out 75 patients). Other side effects (2.67\% or 2 out of 75 patients) noted were headache, increased risk for infection, lethargy, elevated liver function tests, gastrointestinal discomfort, and abdominal pain. Less common ones, which comprised $1.33 \%$ or 1 out of 75 patients, were increased anemia, neutropenia, increased prothrombin time, bone marrow suppression, fatigue, photosensitivity, pancreatitis, and transient worsening of alopecia during the first 6 months of treatment.

\section{DISCUSSION}

Current evidence lacks a proper randomized controlled trial due to the rarity of the extensive forms of alopecia. ${ }^{5}$ Although there was one randomized study by Verma, Gupta, and Khandpur ${ }^{26}$ which compared weekly WAP and BOMP in the treatment of moderate to severe alopecia, only an abstract was obtained for this study, making it ineligible to be included in this systemic review. Nevertheless, its conclusion was similar to that of Sonare and colleagues ${ }^{27}$, stating that 


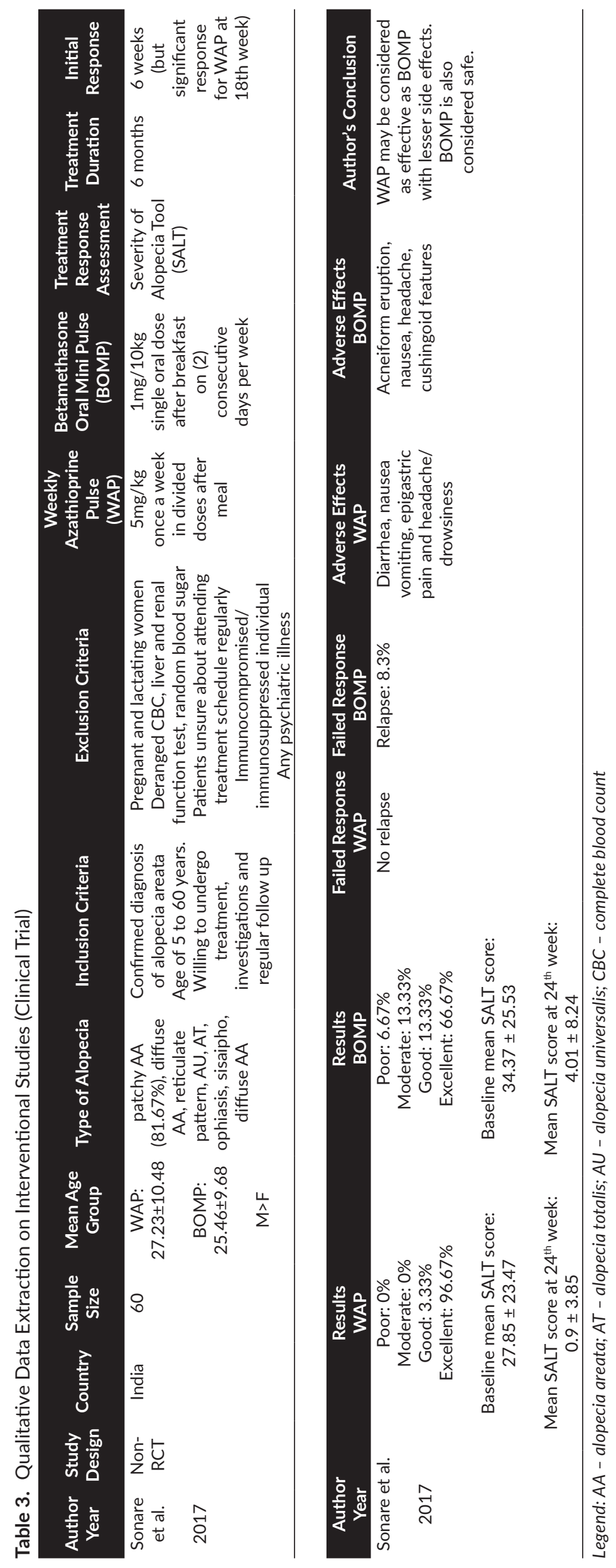


Table 4. Common Adverse Effects Adverse Effect

Frequency Percentage

\begin{tabular}{lcc} 
Abnormal blood count & 1 & \\
$\quad$ anemia & 1.33 \\
$\quad$ neutropenia & 1 & 1.33 \\
$\quad$ mild intermittent leukopenia & 3 & 4.00 \\
unspecified & 1 & 1.33 \\
Increased prothrombin time & 1 & 1.33 \\
Bone marrow suppression & 1 & 1.33 \\
Fatigue & 1 & 1.33 \\
Photosensitivity & 1 & 1.33 \\
Headaches & 2 & 2.67 \\
Infection (unspecified) & 2 & 2.67 \\
Lethargy & 2 & 2.67 \\
Nausea, vomiting & 4 & 5.33 \\
Elevated liver function tests & 2 & 2.67 \\
Gastrointestinal discomfort, abdominal pain & 2 & 2.67 \\
Diarrhea & 3 & 4.00 \\
Pancreatitis & 1 & 1.33 \\
Transient worsening of alopecia & 1 & 1.33 \\
\hline Total (n=75) & 29 & 38.67 \\
\hline
\end{tabular}

WAP and BOMP are equally effective in the treatment of moderate to severe alopecia areata. Adverse effects noted were transient nausea for the WAP group and unspecified corticosteroid-induced outcomes for the BOMP group, thereby concluding that WAP may be a potentially effective and safe alternative to corticosteroids in the treatment of this disease.

Inherent to the type of studies in the observational studies included in this review, it was difficult to prove that hair regrowth was due to azathioprine especially that most of the studies had concomitant steroid treatments for their respective primary autoimmune disease. The case reports did not wait for spontaneous remission since the extent of alopecia of these patients connoted a poor prognosis. ${ }^{38}$ Comparing the time to initial response to that of spontaneous remission, which mostly occurs within 6 months ${ }^{8}$, the early response of the alopecia to azathioprine was from 6 weeks to 4 months. Removal of azathioprine by Vivehanantha and Velangi ${ }^{33}$ led to the relapse of the patient, and subsequent return to azathioprine therapy brought back hair on the scalp of the patient, thereby ruling out that response was merely by chance alone. These observations, albeit case reports and case series, provided compelling evidence to do interventional studies to test the efficacy of azathioprine for alopecia areata.

The study of Farshi et al. ${ }^{25}$ was the most commonly cited pre-post study that showed systemic monotherapy with azathioprine produced clinically relevant improvement in moderate-to-severe alopecia areata. Initial response was noted as early as 3 months, and no relapse was noted for at least 6 months. In contrast, Vañó-Galván et al. ${ }^{36}$ noted two patients who relapsed after 2.5 months of therapy cessation. Nevertheless, the authors agreed with the findings of Farshi and colleagues ${ }^{25}$ that oral azathioprine may be an interesting therapeutic option for adult patients with recalcitrant forms of extensive areata. Because the two studies measured the outcomes differently, this will lead to substantial heterogeneity; thus, it is not possible to do a statistical analysis.

The observational studies and the pre-post studies showed that with azathioprine, the amount of time to hair regrowth is shortened. The next question would be how does this compare to the usual management of severe forms of alopecia areata such as systemic corticosteroids? Sonare et al. ${ }^{27}$ performed a clinical trial illustrating azathioprine to be as effective as oral corticosteroids in treating alopecia areata, with lesser side effects. There was no relapse rate noted with weekly WAP even after 6 months of therapy cessation compared with BOMP, which had 8.3\% relapse. This was significantly lower compared to the findings of Shreberk-Hassidim et al. ${ }^{13}$ in their systematic review of pulse corticosteroid therapy for alopecia areata, which reported a total of $17 \%$ relapse rate. Furthermore, previous studies on pulse corticosteroid therapy for alopecia areata yielded unsatisfactory results for extensive forms of alopecia areata such as $\mathrm{AT}$ and $\mathrm{AU}^{14,15}$, which were similar to that of the findings in the BOMP group of Sonare et al. ${ }^{27}$ as illustrated by the poor and moderate responders at the end of the study.

From the consistent results seen in the observational and interventional studies, it may be concluded that there is fair evidence to recommend azathioprine as a treatment for extensive forms of alopecia areata. Regular laboratory examination is important to monitor adverse effects. Most patients taking the drug would report nausea and vomiting on the first few days of treatment, which would resolve without dose titration. Other forms of GI upset such as diarrhea and abdominal pain were common findings as well. Elevated liver function tests occurred in a few patients while abnormal blood counts and myelosuppression were only seen in $1 \%$ of the population studied. Other side effects reported in the studies but not noted in the BAD guidelines for the safe and effective prescribing of azathioprine ${ }^{11}$, were headache, lethargy, unspecified infection, and transient worsening of alopecia.

A major limitation of this review is the lack of randomized controlled trials available in the literature since the efficacy of azathioprine has only been recently explored in 2010. Furthermore, the population being studied is also rare, making it difficult to come up with a clinical trial that possesses a statistically acceptable sample size. Most of the participants were also adults, making it difficult to extrapolate the data to the pediatric population. Lastly, there are no quality assessment tools to uniformly evaluate the obtained observational and interventional studies for this systematic review.

\section{CONCLUSION}

This is the first systematic review to assess the efficacy and safety of azathioprine for severe forms of alopecia areata. In conclusion, there is emerging evidence on the 
efficacy of azathioprine for the treatment of extensive form of alopecia areata. The drug is well tolerated and has been shown to provide less adverse effects compared to that of systemic corticosteroid therapy. Azathioprine may be considered as a steroid-sparing monotherapy for patients with extensive and steroid-resistant alopecia areata. Weekly complete blood count with platelet count for the first 4 weeks, followed by every 3 months thereafter, must be done due to the lack of TPMT assay locally. ${ }^{11,39}$ Randomized-controlled clinical trials may be pursued to provide more compelling evidence for the efficacy of azathioprine for extensive forms of alopecia areata. A multi-center study may be done to increase the sample size enrolled in the study and to avoid sampling bias.

\section{Disclaimer}

The views expressed in this article are the authors' own and do not reflect the views of the institution.

\section{Statement of Authorship}

All authors approved the final version submitted.

\section{Author Disclosure}

All authors declared no conflict of interest.

\section{Funding Source}

This paper was funded by the authors. No external funding agency.

\section{REFERENCES}

1. Nelson DA, Spielvogel RL. Alopecia areata. Int J Dermatol. 1985;24:26-34.

2. Islam N, Leung PSC, Huntley AC, Eric Gershwin M. The autoimmune basis of alopecia areata: A comprehensive review. Autoimmun Rev. 2015;14(2):81-9.

3. Alkhalifah A, Alsantali A, Wang E, McElwee KJ, Shapiro J. Alopecia areata update. Part I. Clinical picture, histopathology, and pathogenesis. J Am Acad Dermatol. 2010;62(2):177-88.

4. Patterson JW. Diseases of Cutaneous Appendages. In: Weedon's Skin Pathology. 4th ed. Churchill Livingstone-Elsevier; 2016. p. 457-507.

5. Tan E, Tay YK, Goh CL, Giam YC. The pattern and profile of alopecia areata in Singapore - A study of 219 Asians. Int J Dermatol. 2002 Nov;41(11):748-53.

6. Sharma VK. Profile of Alopecia Areata in Nothern India. Int J Dermatol. 1996;35(2):133-6.

7. Messenger AG, McKillop J, Farrant P, McDonagh AJ, Sladden M. British Association of Dermatologists' guidelines for the management of alopecia areata. Br J Dermatol. 2012;166(5):916-26.

8. Walker SA, Rothman S. Alopecia Areata1. J Invest Dermatol. 1950;14(6):403-13.

9. Pascher F, Kurtin S, Andrade R. Assay of $0.2 \%$ Fluocinolone Acetonide Cream for Alopecia Areata and Totalis. Dermatologica. 1970;141(3):193-202. 1970;141(3):193-202.

10. Tosti A, Piraccini BM, Pazzaglia M, Vincenzi C. Clobetasol propionate $0.05 \%$ under occlusion in the treatment of alopecia totalis/universalis. J Am Acad Dermatol. 2003;49(1):96-8.

11. Meggitt SJ, Anstey A V., Mohd Mustapa MF, Reynolds NJ, Wakelin S. British Association of Dermatologists' guidelines for the safe and effective prescribing of azathioprine. $\mathrm{Br} \mathrm{J}$ Dermatol. 2011;165(4):711-34
12. Dainichi T, Kabashima K. Alopecia areata: What's new in epidemiology, pathogenesis, diagnosis, and therapeutic options? J Dermatol Sci. 2017;86(1):3-12.

13. Shreberk-Hassidim R, Ramot Y, Gilula Z, Zlotogorski A. A systematic review of pulse steroid therapy for alopecia areata. J Am Acad Dermatol. 2016;74(2):372-374.e5.

14. Staumont-Sallé D, Vonarx M, Lengrand F, Segard M, Delaporte E. Pulse corticosteroid therapy for alopecia areata: long-term outcome after 10 years. Dermatology. 2012;225(1):81-7. doi: 10.1159/000341523.

15. Açıkgöz G, Özmen İ, Çayırlı M, Yeniay Y, Köse O. Pulse methylprednisolone therapy for the treatment of extensive alopecia areata. J Dermatolog Treat. 2014;25(2):164-6.

16. Spano F, Donovan JC. Alopecia areata: Part 2: treatment. Can Fam Physician. 2015;61(9):757-61.

17. Carnahan MC, Goldstein DA. Ocular complications of topical, peri-ocular, and systemic corticosteroids. Curr Opin Ophthalmol. 2000;11(6):478-83.

18. Vañó-Galván S, Hermosa-Gelbard Á, Sánchez-Neila N, MiguelGómez L, Saceda-Corralo D, Rodrigues-Barata R, et al. Pulse corticosteroid therapy with oral dexamethasone for the treatment of adult alopecia totalis and universalis. J Am Acad Dermatol. 2016;74(5):1005-7.

19. Wasserman D, Guzman-Sanchez DA, Scott K, Mcmichael A. Alopecia areata. Int J Dermatol. 2007;46(2):121-31

20. Downs AM, Lear JT, Kennedy CT. Anaphylaxis to intradermal triamcinolone acetonide. Arch Dermatol. 1998;134(9):1163-4.

21. Laing ME, Fallis B, Murphy GM. Anaphylactic reaction to intralesional corticosteroid injection. Contact Dermatitis. 2007;57(2):132-3.

22. Samrao A, Fu JM, Harris ST, Price VH. Bone mineral density in patients with alopecia areata treated with long-term intralesional corticosteroids. J Drugs Dermatol. 2013;12(2):e36-40.

23. Kurosawa M, Nakagawa S, Mizuashi M, Sasaki Y, Kawamura M, Saito M, et al. A Comparison of the Efficacy, Relapse Rate and Side Effects among Three Modalities of Systemic Corticosteroid Therapy for Alopecia Areata. Dermatology. 2006; 8574:361-5.

24. Renert-Yuval Y, Guttman-Yassky E. The Changing Landscape of Alopecia Areata: The Therapeutic Paradigm. Adv Ther. 2017;34(7):1594-609.

25. Farshi S, Mansouri P, Safar F, Khiabanloo SR. Could azathioprine be considered as a therapeutic alternative in the treatment of alopecia areata? A pilot study. Int J Dermatol. 2010;49(10):1188-93.

26. Verma K, Gupta P, Khandpur S. Comparison of effectiveness of weekly azathioprine pulse (WAP) and betamethasone oral mini pulse (BOMP) in the treatment of moderate to severe alopecia areata. J Am Acad Dermatol. 2015;72(5):AB112.

27. Sonare D, Seervi R, Vats G, Meena M, Kataria R, Jain V. A Comparative Study of Safety and Efficacy of Betamethasone Oral Mini Pulse (Bomp) and Weekly Azathioprine Pulse (Wap) in the Treatment of Alopecia Areata. World J Pharm Med Res. 2017;3(4):47-54.

28. Elion GB. The Pharmacology of Azathioprine. Ann New York Acad Sci. 1993;685(1):401-7.

29. Patel AA, Swerlick RA, McCall CO. Azathioprine in dermatology: The past, the present, and the future. J Am Acad Dermatol 2006;55(3):369-89.

30. Maltzman JS, Koretzky GA. Azathioprine: old drug, new actions. J Clin Invest. 2003;111(8):1122-4.

31. Thiese MS. Observational and interventional study design types; an overview. Biochem Medica. 2014;24(2):199-210.

32. Goddard CJR, August PJ, Whorwell PJ. Alopecia totalis in a patient with Crohn's disease and its treatment with azathioprine. Postgrad Med J. 1989;65(761):188-9.

33. Vivehanantha S, Velangi S. Azathioprine: A therapy to be considered in alopecia universalis. J Am Acad Dermatol. 2014;70(5):AB89.

34. Bala RH, Chong AH. A case series of 10 patients with steroid resistant alopecia areata successfully treated with azathioprine. Australas J Dermatol. 2016;57:69.

35. Shohdy KS, Rashad W, Elmeligui A. Alopecia universalis associated with ulcerative colitis and the role of azathioprine. Middle East J Dig Dis. 2018;10(1):50-4. 
36. Vañó-Galván S, Hermosa-Gelbard Á, Sánchez-Neila N, MiguelGómez L, Saceda-Corralo D, Rodrigues-Barata R, et al. Treatment of recalcitrant adult alopecia areata universalis with oral azathioprine. J Am Acad Dermatol. 2016;74(5):1007-8.

37. Olsen EA, Hordinsky MK, Price VH, Roberts JL, Shapiro J, Canfield $\mathrm{D}$, et al. Alopecia areata investigational assessment guidelines-Part II. J Am Acad Dermatol. 2004;51(3):440-7.
38. Tosti A, Bellavista S, Iorizzo M. Alopecia areata: A long term followup study of 191 patients. J Am Acad Dermatol. 2006;55(3):438-41.

39. Wise M, Callen JP. Azathioprine: A guide for the management of dermatology patients. Dermatol Ther. 2007;20(4):206-15.

The Acta Medica Philippina is now accepting limited advertising for its front and back cover (colored), as well as for available spaces in some of its pages, as appropriate. For inquiries and submission of proposals, please e-mail us at actamedicaphilippina@yahoo.com 